\title{
Transatlantische Karrieren und transnationale Leben: zum Verhältnis von Migrantenbiographien und transnationaler Geschichte
}

\author{
Jan Logemann
}

\section{Einleitung}

Der Soziologe Paul Lazarsfeld hatte nicht nur eine transatlantische Karriere, er führte ein transnationales Leben. Immer wieder kreuzte er den Atlantik und war sowohl in Wien als auch in New York lange heimisch. Er war ein transatlantischer Mittler und hinterließ Spuren, die weit über seine eigene Biographie hinausweisen. Der amerikanische Gesellschaftstheoretiker James Coleman schrieb 1976 in einem Nachruf, Lazarsfelds Forschung an der Columbia University hätte „für eine geraume Zeit die führende Kraft in der amerikanischen Soziologie" ausgemacht. Für Europäer, so Coleman, sei seine Arbeit zudem der Inbegriff für die amerikanische Soziologie schlechthin gewesen. Es sei ein Paradoxon, resümierte der Nachruf, dass die ,amerikanische" Soziologie, welche nach dem Zweiten Weltkrieg Europa so stark beeinflusst habe, zu einem beachtlichen Teil durch einen Mann geprägt worden sei, der in Mitteleuropa geboren und ausgebildet wurde (Coleman 1976: 7). Verkürzt gesagt hatte der „Europäer“ Lazarsfeld in Amerika die empirische Soziologie mitgeprägt und diese dann nach dem Krieg als etwas „Amerikanisches“ nach Europa re-exportiert und somit zu einem reziproken, transnationalen Gesellschaftsaustausch beigetragen. ${ }^{1}$ Diese transatlantische Karriere des Emigranten Lazarsfeld, der Anfang der 1930er Jahre zuerst von Wien in die USA überwechselte, soll hier als ein Beispiel für jene Form von grenzüberschreitenden Migrantenbiographien herangezogen werden, mit deren Bedeutung für das Schreiben transnationaler Geschichte ich mich im Folgenden auseinandersetzen möchte.

Wie misst man ein „transnationales Leben“? Wie kommen wir von Migrantenbiographien $\mathrm{zu}$ weitergreifenden Fragen transnationaler Geschichte? Nachrufe sind sicherlich, wie viele biographische Dokumente, eine problematische Quelle. ${ }^{2}$ Es ist charakteristisch für das Genre, dass positive Bewertungen von Lebensleistungen und eine Überhöhung der Bedeutung von Persönlichkeiten einhergehen mit einem oft selektiven Blick des jeweiligen Nachrufschreibers. Zudem wurde und wird nur einem sehr begrenzten Kreis von Personen die Ehre eines veröffentlichten Nachrufs zuteil es handelt sich dabei meist um eine Gruppe von Personen, die wie Lazarsfeld einer

1 Zum transatlantischen Austausch in den Sozialwissenschaften zu dieser Zeit siehe Christian Fleck (2007).

2 Zur Problematik biographischer Quellen unter dem Aspekt der Inszenierung siehe Thomas Etzemüller (2012). 
gesellschaftlichen Elite zugerechnet werden können. In der Tat soll es hier vor allem um die „transnationalen Leben“ von Funktionseliten gehen, deren Leben und Wirken sich aufgrund von Bildung oder anderen Ressourcen oft sehr stark von den Lebenswirklichkeiten der großen Mehrheit von Migranten im zwanzigsten Jahrhundert unterschied. Mit dieser Einschränkung möchte ich Colemans Beobachtungen über Lazarsfelds transatlantisches Wirken dennoch als einen Ausgangspunkt dafür nehmen, allgemeiner über das Potential des biographischen Blicks für das boomende Feld der transnationalen Geschichtsschreibung zu reflektieren.

Die Analyse transnationaler Leben bietet Einblicke in globale Wissenstransfers und in die Elitennetzwerke, die neue Formen von „Globalität“ trugen. Im Forschungsprojekt Transatlantic Perspektives: Europa in den Augen europäischer Einwanderer in den USA verfolgten wir transnationale Austauschprozesse im transatlantischen Raum um die Mitte des zwanzigsten Jahrhunderts in den Bereichen von Sozialwissenschaft und Stadtplanung sowie von Wirtschaft und Massenkonsum, die für Vorstellungen gesellschaftlicher „Modernität“ jener Zeit von herausgehobener Bedeutung waren. ${ }^{3}$ Wenngleich es sich nicht explizit um ein biographisches Projekt handelte, so nutzten wir individuelle Leben und Karrieren dennoch als ein Prisma, um transnationalen Prozessen nachzuspüren. Damit können wir zu einer neu-belebten Biographieforschung beitragen, die ein erweitertes Verständnis des Biographischen propagiert. Dieses schließt eine „Dezentrierung“ des Individuums und ein Verständnis von dessen Konstruiertheit ebenso mit ein wie die Pluralität verschiedener Rollen, von denen sich das Projekt auf die professionelle Rolle konzentriert hat. Zudem erlebte das Feld eine Erweiterung jener Gruppe, die zum Subjekt von biographischen Betrachtungen wird. Genau wie die Biographik schon längst nicht mehr die sprichwörtlichen „großen, weißen Männer“ zum Zentrum ihrer Untersuchung macht oder den Anspruch erhebt, Leben in ihrer Totalität oder gar ihrem Wesenskern darzustellen, so steht sie auch nicht mehr allein im Dienste einer national-zentrierten Geschichtsschreibung. ${ }^{4}$ Es bietet sich daher Raum, nach den Karrieren von Migranten zu fragen, deren Namen zum Teil nur noch Spezialisten ein Begriff sind und deren ,transnationale Leben" dennoch entscheidend zu grenzüberschreitenden Transfers und zu globaler Vernetzung um die Mitte des zwanzigsten Jahrhunderts beigetragen haben.

Transfers und die Entwicklung verschiedener Formen globaler Vernetzung sind mittlerweile ein zentrales Forschungsfeld der Geschichtswissenschaft. Biographische Ansätze, so möchte ich hier argumentieren, bieten eine Möglichkeit, die Forderung nach immer stärkerer Transnationalität in der Geschichtsschreibung zu operationalisieren und empirisch zu erfüllen. Als „transnationale Leben“ verstehe ich dabei jene, die durch ein hohes Maß grenzüberschreitender Mobilität längerer Dauer geprägt waren und daher Formen transkultureller Adaption verlangten. ${ }^{5}$ Eine solche Definition schließt auch manche „Reisende“ mit ein, zielt aber vor allem auf eine in sich sehr

3 Das vom Bundesministerium für Bildung und Forschung (BMBF) geförderten Projekt lief zwischen 2010 und 2014 am Deutschen Historischen Institut in Washington, vgl. Logemann/Joch/Ludwig/ Narayan/Reiterer (2011), 85-99. Weitere Informationen: www.transatlanticperspectives.org (zuletzt besucht am 1.6.2016).

4 Zur Entwicklung der Biographik im transatlantischen Kontext siehe Volker Depkat (2014) und allgemein Christian Klein (2009).

5 Zum Begriff der transnationalen Leben vgl. Deacon/Russell/Woollacott (2010). 
heterogene Gruppe von Kurz- und Langzeitmigranten ab. ${ }^{6}$ Der biographische Blick auf solche Migranten erlaubt es, bestimmte Aspekte von Transfer- und Austauschprozessen wenigstens in Teilbereichen konkret herauszuarbeiten: (1.) die Bedeutung von Migranten und deren physischer Präsenz für grenzüberschreitenden und reziproken Wissenstransfer, (2.) die Bedeutung von institutionellen oder persönlichen Netzwerken für die Verstetigung und Nachhaltigkeit von Austauschprozessen sowie (3.) die Zentralität von aktiven Übersetzungsleistungen bei der Lokalisierung und Adaption von Wissen und Praktiken.

Zwei Typen der Narration von „transnationalen Leben“ sind für die transnationale Geschichtsschreibung von besonderem Interesse: einerseits „transgressive Biographien“, die bestehende (nationalstaatliche) Ordnungen herausfordern, und andererseits „transnationale Mittler“, die als Brückenbauer zwischengesellschaftlichen Transfer und Austausch beförderten. Diese beiden Typen sollen zunächst generell in das Verhältnis von transnationaler Geschichtsschreibung und Biographik eingeordnet werden. Die Rolle von Mittlern im Kontext der transatlantischen Beziehungen im zwanzigsten Jahrhundert stand dabei im Zentrum des Transatlantic Perspectives-Projekts, auf das ich im Folgenden eingehe. Die Aspekte von reziprokem Wissenstransfer, Netzwerkbildung, und Übersetzungsleitung möchte ich dann an konkreten biographischen Beispielen aus diesem Forschungszusammenhang darstellen. Abschließend will ich den gewählten Ansatz kurz problematisieren und nach dem kritischen Potential von biographischen Zugriffen fragen, die oft sehr glatten Erzählungen globaler Vernetzung und grenzüberschreitenden Austauschs zu brechen.

\section{Transnationale Geschichtsschreibung und Biographik}

Transnationalität war in den vergangenen zwanzig Jahren eines der großen Buzzwords der Geschichtswissenschaft beiderseits des Atlantiks. Unter dem Eindruck allgegenwärtiger Globalisierungstendenzen, die man zu historisieren suchte, und von Nationalstaaten, deren Bedeutung zu schwinden schien, lag der Fokus dieser Forschungsperspektive zunächst auf zwischenstaatlichen Institutionen, zivilgesellschaftlichen Bewegungen und wirtschaftlichen Zusammenhängen, die grenzübergreifend wirkten. Multinationale Firmen, internationale Bewegungen und supranationale Organisationen standen dabei jedoch eher im Mittelpunkt der Forschung als einzelne Individuen und deren Lebensgeschichten. Unter den knapp 500 Einträgen des 2007 erschienen Palgrave Dictionary of Transnational History finden sich gerade mal fünfzehn Namenseinträge und nur sechs wirkliche biographische Artikel (Iriye/Saunier 2009). ${ }^{7}$ Dabei kann eine kontextorientierte transnationale Biographik nicht nur der Akzentuierung und Erweiterung bestehender Diskurse der transnationalen Geschichtsschreibung dienen, sondern auch neue Perspektiven auf den Ablauf und auf die Mikroebene grenzüberschreitender Austauschprozesse eröffnen.

6 Der Migrationsbegriff hat sich in der neueren Forschung deutlich erweitert, wobei die Grenzen zum Mobilitätsbegriff teilweise unscharf bleiben. Vgl. Harzig/Hoerder/Gabaccia (2009).

7 Die Mehrzahl der Namenseinträge sind reine Verweise wie zum Beispiel Mohandas Ghandi auf nonviolence oder bei Aletta Jacobs zu women's movement. Wirkliche biographische Skizzen finden sich nur zu Marcus Garvey, Ernesto Guevara, Harry von Kessler, Paul Otlet, Saba George Shiber und zur Familie Hammarskjöld. 
Der Blick auf die großen Fragen globalhistorischer Zusammenhänge führte in den frühen Jahren der Diskussion um transnationale Geschichte zum Teil zu einer mangelnden Konkretheit; der Untersuchungsgegenstand blieb im Versuch der theoretischen Bestimmung oft wenig greifbar. Transnationalität galt ab Mitte der 1990er Jahre in der amerikanischen und mit gewisser zeitlicher Verzögerung dann auch in der deutschen Historiographie als das neue Paradigma der Geschichtsschreibung, welches den Nationalstaat als fundamentale Analyseeinheit historischer Erzählungen ablösen oder wenigstens transzendieren sollte (Tyrrell 1991). In zahlreichen Debattenbeiträgen ging es dabei zunächst um Begriffsklärungen und Abgrenzungen etwa gegenüber der New International History (als einer erweiterten Form der Diplomatiegeschichte), der traditionellen historischen Komparatistik oder der noch weiter greifenden, gelegentlich auch unter dem verwandten Begriff der histoire croisée firmierenden, Verflechtungsgeschichte (vgl. u.a. Tyrrell 2009 und Patel 2003). Im deutschen Fall bot sich ein Anknüpfen an die in den 1980er Jahren entwickelte Transfergeschichte, die ursprünglich auf die Analyse von inner-europäischem Gesellschaftsaustausch abzielte, an (vgl. Middell 2000). Allgemein ergaben sich zudem immer größere Schnittmengen mit den Ansätzen der neueren Globalgeschichte, die im Gegensatz zur Globalisierungsgeschichte oder der älteren Weltgeschichte weltumspannende Metanarrative eher vermeidet und regionale Verflechtungen in globalen Kontexten untersucht. ${ }^{8}$ Jenseits der methodischen Bestimmung blieb die praktische Umsetzung und Operationalisierung des Rufs nach transnationaler Geschichte allerdings zunächst ein selten eingelöstes Versprechen.

Eine präzise Bestimmung der mit dem neuen Paradigma verbundenen inhaltlichen Forschungsprogramme fiel zunächst schwer. In den USA war das Konzept der transnationalen Geschichte zuerst eng mit den Borderlands Studies verbunden, die sich mit den Dimensionen der historischen Verflechtung von Grenzregionen auseinandersetzten (z. B. Thelen 1992). Hier ist der Blick auf die vielfältigen Kontakte und Verbindungen gerichtet, die über nationalstaatliche Grenzen hinweg regionale Räume - etwa zwischen den USA und Mexiko aber ähnlich auch im innereuropäischen kleinen Grenzverkehr - konstruierten. Andere, wie der Historiker Thomas Bender, sahen transnationale Geschichte dagegen zweitens vor allem als eine Agenda zur „Globalisierung“ der amerikanischen Geschichte. Entgegen Narrativen eines American Exceptionalism verwiesen Thomas Bender (2006) und Ian Tyrell (2007) auf die größeren globalen Zusammenhänge, in denen sich amerikanische Geschichte etwa im Bürgerkrieg oder im New Deal abspielte. Auch in der deutschen Geschichtsschreibung hat sich die Ansicht weitgehend durchgesetzt, dass ,nationale“ Geschichte nur in europäischen und globalen Kontexten sinnvoll erzählt werden kann (vgl. beispielhaft Conrad/Osterhammel 2004; Osterhammel 2009).

Eine dritte Konzeption von transnationaler Geschichte stellt multidirektionale Austauschbeziehungen und Verflechtungen zwischen Gesellschaften ins Zentrum der Untersuchung. Bei diesem Zugriff, der auch für das hier zugrundeliegende Transatlantic Perspectives-Projekt erkenntnisleitend war, geht es um grenzübergreifende Ströme (Flows) von Waren, Menschen und Ideen. Für den transatlantischen Raum hat der Historiker Daniel Rodgers hier Pionierarbeit geleistet, der den europäischamerikanischen Austausch über soziale Probleme der Moderne unter Wissenschaft-

8 Zum Verhältnis zur Globalgeschichte vgl. Matthew Hilton und Rana Mitter (2013). 
lern, Intellektuellen und Politikern um die Wende zum zwanzigsten Jahrhundert untersucht hat (Rodgers 1998). Neben Studien zur Geschichte globaler Ideentransfers gibt es mittlerweile zahlreiche Arbeiten zu transnationalen Wirtschaftsprozessen sowie zu globalen Migrationsbewegungen. ${ }^{9}$ Dabei erscheinen Austauschprozesse weniger als Teil eines allumfassenden, irreversiblen oder zielgerichteten Globalisierungsprozesses. Vielmehr geht es um eine historische Annäherung an Formen der gesellschaftlichen Verflechtung und der wechselseitigen Beeinflussung zwischen verschiedenen Weltregionen besonders unter den Bedingungen globaler Markt- und (post)kolonialer Machtstrukturen (vgl. Rosenberg 2012).

Diese auf Formen des Transfers fokussierte Konzeption transnationaler Geschichte lässt sich gut mit einem in der europäischen Historiographie prominenten Interesse an überstaatlichen und nicht-staatlichen Institutionen und Organisationen verbinden. Durch sie geschaffene formelle und informelle Rahmen machten Transfers oft erst möglich oder halfen dabei, sie zu verstetigen. Dies trifft zum einen auf politische Organisationen $\mathrm{zu}$, wie etwa den Völkerbund in der Zwischenkriegszeit oder die OECD in den Jahrzehnten nach dem Zweiten Weltkrieg (vgl. u.a. Herren 2009 und Schmelzer 2012). Aber auch informelle Netzwerke von Experten und Spezialisten (epistemische Gemeinschaften) bzw. deren internationale Organisationen haben die Aufmerksamkeit dieser Art von Geschichtsschreibung auf sich gezogen, wie das Beispiel der Berufsgruppe von Architekten und Stadtplanern und der transnationalen Architektenverbindung CIAM zeigt (vgl. u.a. Domhardt 2011 und Wagner 2014). Wie wichtig der institutionelle Rahmen für grenzüberschreitende Transfers von Personen oder Wissen sein kann, zeigt auch die wachsende Forschung zu den großen amerikanischen Stiftungen wie der Carnegie Endowment und den Ford- und Rockefeller-Stiftungen, die transnationale Austauschprozesse massiv finanziell beförderten und aufrechterhielten (vgl. Gemelli/MacLeod 2003; Krige/Rausch 2012). Seit dem späten neunzehnten Jahrhundert bildete sich eine zunehmend institutionalisierte und immer engmaschigere „transnationale Sphäre“ von Expertennetzwerken heraus, die von solchen Organisationen getragen wurde (vgl. Rodogno/Struck/Vogel 2015).

Genauso wie der Kontext der Kolonialgeschichte für die Untersuchung von globalen Transfers im neunzehnten Jahrhundert konstitutiv ist, stellt für weite Strecken des zwanzigsten Jahrhunderts die Frage nach amerikanischen Einflüssen (etwa im Sinne einer „Amerikanisierung“) einen entscheidenden Antrieb für Forschungen zu transnationalen Flows dar (de Grazia 2005). Im Zusammenhang des Kalten Krieges verbanden sich grenzüberschreitende Netzwerke häufig mit politischer Einflussnahme und hegemonialen Machtansprüchen, wie sich am Beispiel des Congress for Cultural Freedom zeigen lässt (Scott-Smith 2010; Hochgeschwender 1998). Gleichzeitig waren aber auch die neuen sozialen Bewegungen der Nachkriegsjahrzehnte und jene, die wie die Friedensbewegung die Ordnung des Kalten Krieges in Ost wie West herausforderten, stark transnational vernetzt. Gerade mit Blick auf diese Bewegungen und Institutionen hat die Forschung der vergangenen zwanzig Jahre einiges zur empirischen Gehaltfüllung von „Transnationalismus“ geleistet (Klimke 2012).

Eine biographische Perspektive bietet nun die Möglichkeit, noch direkter auf die Mikroebene transnationaler Prozesse vorzudringen. Gerade der institutionenge-

9 Zur Geschichte globaler Wirtschaftsbeziehungen aus deutscher Sicht beispielhaft Torp (2005) und Rischbieter (2011). Zur Migrationsgeschichte vgl. u.a. Hoerder (2002) und McKeown (2008). 
schichtliche Ansatz läuft manchmal Gefahr, eine abstrakte und unkontextualisierte transnationale Sphäre zu konstruieren, deren grenzüberschreitende Diskurse scheinbar in einem luftleeren ,globalen“ Raum schweben. Die Untersuchung transnationaler Netzwerke und Institutionen muss daher rückgebunden bleiben an historische Entwicklungen in nationalen, regionalen und lokalen Kontexten. Immer wieder gilt es zu zeigen, wie sich die transnationale Ebene sinnvoll mit historischen Erfahrungen auf anderen Ebenen verbinden lässt. So gelingt es zum Beispiel Volker Berghahn, sich der Komplexität und Wirkungsmacht transatlantischer Netzwerke der Nachkriegszeit durch das transnationale Leben des Amerikaners Shepard Stone zu nähern (Berghahn 2004). Petra Kellys transatlantische Karriere ist in ähnlicher Weise herangezogen worden, um Austauschprozesse über den Atlantik hinweg in einer neuen Form von zivilgesellschaftlicher Gegenöffentlichkeit zu konkretisieren (Milder 2010). Erst der Blick auf die Akteursebene legt dabei die genauen Bedingungen von grenzüberschreitenden Transfers frei, einschließlich der Adaptionen, der Veränderungen, der hybriden Neuschöpfungen sowie auch der fehlgeschlagenen Vermittlungen, die zentrale Bestandteile dieser Prozesse sind.

Transfer und Vernetzung bedürfen der Akteure, und hier kann die transnationale Geschichtsschreibung sinnvoll an die jüngere Migrationsgeschichte anknüpfen. Die historische Migrationsforschung hat in den vergangenen Jahrzehnten ihre eigene transnationale Wende gemacht. Galten lange die Paradigmen der Integration und der Assimilierung in die Aufnahmegesellschaft als erkenntnisleitend für diesen Forschungszweig, so rückten nun die komplexen Pfade der Migration, die Multidirektionalität von Wanderungsbewegungen, und die anhaltenden Wechselbeziehungen zwischen Ziel- und Herkunftsregionen der Migranten in den Mittelpunkt migrationshistorischer Studien (z. B. Gabaccia 2010). Der Typus des modernen „Transmigranten“, dessen Leben durch ein ständiges Hin-und-Her-Wandern und die gleichzeitige Verankerung in mehreren Gesellschaften charakterisiert ist, wird in seiner historischen Dimension erkannt (vgl. Portes 2000). Die Leben solcher Transmigranten sind nicht nur „transnationale Leben“ par excellence, sie sind zudem geprägt von Momenten und Orten kultureller Hybridität. ${ }^{10}$ Im Dazwischen, den transnationalen Zwischenräumen der Migration, entstehen kulturelle Neuerungen, die ihre Spuren sowohl in Entsende- wie auch in Aufnahmegesellschaften hinterlassen und dabei die Grenzen zwischen ihnen teilweise verwischen. Mit Blick auf die Sozialisation von Transmigranten in verschiedenen Kulturräumen und der Notwendigkeit, ständig zwischen kulturellen Kontexten zu wechseln, sprechen Migrationshistoriker daher von Transkulturalität und von transkultureller Lebensführung (Hoerder 2005).

Ein weiteres Merkmal der neuen historischen Migrationsforschung ist es, dass sie individuellen Lebensläufen zunehmend Aufmerksamkeit schenkt. Entgegen einer rein quantitativen Analyse von Massenmigrationsbewegungen gibt es ein wiedererwachtes Interesse an einzelnen Migrationswegen, welche die Komplexität von Wanderung und kulturellen Austauschprozessen offenlegen können. Hier kann der biographische Blick vermitteln zwischen der Frage nach Strukturen im Sinne der traditionellen Sozialgeschichtsschreibung und nach der Agency, also der Handlungsmacht, einzelner Akteure (vgl. Berghahn/Lässig 2008). Dies gilt besonders für neue Forschungen zur

10 Grundlegend zum Konzept der Hybridität und zu Formen transkulturellen Austausches durch globale Migration Bhabha (1994). 
Elitenmigration, wie etwa in den Arbeiten der Historikerin Nancy Green, die jüngst die Geschichte amerikanischer Expatriates im Frankreich der Zwischenkriegszeit untersuchte (Green 2014). Geschäftsleute, Intellektuelle, Diplomaten und Professionals gehören in diese Gruppe der elite migrants, deren in vieler Hinsicht exzeptionelle Migrationserfahrung lange aus dem Blickfeld der sozialhistorischen Migrationsgeschichte gefallen war (vgl. Fechter/Walsh 2010). ${ }^{11}$ Studien zur Elitenmigration tauschen nun offensiv den Blick auf die „breite Masse“ der Migranten gegen ein Interesse an Transferprozessen und an transkulturellen Wandlungen ein, welche sich schon quellentechnisch oft am leichtesten bei gesellschaftlichen Funktionseliten nachverfolgen lassen.

Dabei öffnet sich die Migrationsgeschichte dem traditionell eher geistesgeschichtlich geprägten Feld der Geschichte von Emigration und Exil, also von Formen der Zwangsmigration, bei deren Untersuchung schon lange nach transnationalen Transfers durch prominente Wissenschaftler und Intellektuelle gefragt wurde (vgl. Unger 2009). Biographische Ansätze haben in der Emigrationsgeschichte immer eine große Rolle gespielt, und auch diese Forschung hat sich in den vergangenen Jahren zunehmend Fragen von Transnationalität und Transkulturalität angenommen. So lässt sich etwa das Konzept kultureller Hybridität durchaus sinnvoll mit der Erfahrung europäischer Emigranten in den USA verbinden, da grenzüberschreitende Wissenstransfers oft Neues schufen und auch die Aufnahmegesellschaft mit umformten (Krohn 2009). Entgegen dem überkommenen Bild eines Brain-Drain durch Emigration und Exil verweisen viele Emigrationsforscher darüber hinaus auf die Bedeutung von transnationaler Wissenszirkulation durch Elitenmigration im zwanzigsten Jahrhundert (BrainCirculation), von der in der Regel auch die Entsendegesellschaften langfristig profitierten (vgl. Dittmar Dahlmann/Reith 2008).

Die Rolle von Transfers durch Elitenmigration lässt sich sehr gut am Beispiel von Unternehmern mit Migrationshintergrund studieren (Berghoff/Fahrmeir 2013). Die Unternehmensgeschichte, die lange im Strukturwandel von Großunternehmen ihren Hauptuntersuchungsgegenstand sah, hat sich in der jüngeren Vergangenheit vermehrt wieder der Bedeutung von Unternehmern als Akteuren zugewandt. Dabei geht es gerade bei Familienunternehmen auch um die personellen Netzwerke, die sich zwischen Unternehmerfamilien und ihrem sozialen Umfeld entspannen. Bei familiengeführten Multinational Enterprises waren diese Netzwerke schon lange grenzüberschreitend und stellten den transnationalen Austausch von Wissen und Waren, von Personal und Kapital sicher (Lubinski/Fear/Pérez 2013). Ein umfangreiches biographisches Projekt zur Geschichte von Immigrant Entrepreneurs am Deutschen Historischen Institut in Washington sucht derzeit ganz explizit nach verschiedenen Formen des Wissens- und Technologietransfers durch eingewanderte Unternehmer und kontextualisiert diese Elitenmigranten und ihre Familien in transnationalen Räumen zwischen deutscher Entsende- und amerikanischer Aufnahmegesellschaft (Berghoff/Spiekermann 2010; Pertilla/Spiekermann 2014). In den Biographien deutschstämmiger Brauer, Möbelfabrikanten oder Computerentwickler finden sich konkrete Beispiele transnationaler Wissenszirkulation und eine Mikroperspektive auf die

11 Eine gewisse Ausnahme bilden Elitemigranten im kolonialen Kontext, siehe z.B. Habermas/Conrad (2010). 
grenzüberschreitenden Ströme von Waren, Menschen und Kapital im atlantischen Raum der Neuzeit.

Solche „transnationalen Leben“ und die Lebensgeschichten dieser Migranten sind in den vergangenen Jahren verstärkt in den Blick biographisch interessierter, transnationaler Forschung geraten. In Bänden zu transnational lives und ,globalen Lebensläufen“ finden sich biographische Skizzen von Händlern und Entdeckern, von Kolonialbeamten und internationalen Künstlern, aber auch von Aktivisten und Flüchtlingen, die einen akteurszentrierten Blick auf Globalgeschichte ermöglichen sollen (Deacon/Russell/Woolacott 2006). ${ }^{12}$ Der amerikanische Historiker Matthew Pratt Guterl schrieb jüngst in einem Kommentar zum Forum Transnational Lives in the Twentieth Century im American Historical Review, dass der Fokus auf individuelle Lebenswege die Möglichkeit verspricht, den Blick auf große Fragen von Nation, Welt und Trans-Nation für die Forschung handhabbar zu machen und sinnvoll einzugrenzen (Guterl 2013). Mehr als nur bestehendes historisches Wissen transnational zu „erweitern“ oder neu zu lesen, so fordert Guterl, sollte die Erforschung transnationaler Leben auch dazu dienen, vertraute historische Narrative in Frage zu stellen. Gerade mit Blick auf historische Periodisierungen könne eine grenzüberschreitende biographische Perspektive neue Kontinuitäten und Brüche zu Tage fördern. ${ }^{13}$ Eine Möglichkeit, solche Forderungen nach neuen Narrativen einzulösen, kann darin bestehen, eurozentrische Perspektiven auf globale Prozesse durch die Inklusion subalterner Stimmen aufzubrechen, wie postkoloniale Arbeiten zum Kosmopolitismus dies nun versuchen (vgl. besonders Deacon/Russell/Woolacott 2010).

Die Figur des Kosmopoliten, der in seiner oft widersprüchlichen Identität nationale und kulturelle Grenzen überwindet und sich Zuordnungen von „Eigenen“ und „Anderen" zu entziehen sucht, hat zu Beginn des einundzwanzigsten Jahrhunderts starken Zuspruch in der Forschung erfahren (vgl. Sluga/Horne 2010). ${ }^{14}$ Dabei geht es nicht mehr nur um Intellektuelle und deren internationale Vernetzung, Selbstreflexionen und Vorstellungen einer neuen Weltordnung wie im Stereotype des ,cosmopolitan thinker: a European man, a ,continental' of the Jewish diaspora who knew through family experience the true meaning of statelessness and brought this to bear on his conceptual thinking“ (Horne 2010: 420). Homi Bhabha hat solche Arten eines privilegierten global cosmopolitanism herrschender Eliten unterschieden von einem vernacular cosmopolitanism einer breiten Masse von Transmigranten und diasporischen Minderheiten, die sehr andere, aber deshalb nicht weniger kosmopolitische, globale Leben führen (Bhabha 2004). Beide von Bhabha genannten Formen kosmopolitischer Lebensführung bieten der transnationalen Geschichte wichtige Anknüpfungspunkte und verweisen implizit auf zwei unterschiedliche Typen transnationaler Leben.

Der erste Typ sind die von Madeleine Herren so genannten ,transgressiven Biographien“. Hiermit meint Herren Lebensverläufe, die herkömmliche Rahmen sprengten und dabei sowohl nationalstaatliche Grenzen überschritten als auch bestehende soziale und kulturelle Normen herausforderten, aufbrachen und manchmal an diesen

12 Gleichzeitig hat auch die Biographik ein Interesse an Transnationalität entwickelt und fragt danach, wie diese verändernd auf das biographische Subjekt wirkt (vgl. Schweiger 2012).

13 Guterl verweist dabei auf das Beispiel der Civil Rights Era der 1950er und 1960er Jahre, die sich durch biographische Betrachtungen strecken ließe (Guterl 2013: $135 \mathrm{f}$.).

14 Zur Definition des Kosmopoliten auch Schweiger (2012), der sich dabei an Ulrich Beck orientiert. 
scheiterten (Herren 2005). Transgressive Biographien von politischen Aktivisten können etwa auf transnationale Gegenöffentlichkeiten und Zivilgesellschaften hinweisen oder - wie im Fall vieler Migrantengruppen - auf ganz alltägliche Weise gesellschaftliche und staatliche Ordnungsentwürfe in Frage stellen. Die Lebensentwürfe und Erfahrungen solcher „Grenzgänger“ eignen sich nicht nur besonders gut, die Brüche in nationalen Narrativen aufzuzeigen, sondern sensibilisieren uns gleichzeitig für die historische Konstruiertheit staatlicher, gesellschaftlicher und kultureller Grenzen. ${ }^{15}$ Jedoch waren nicht alle transnationalen Leben in diesem Sinne „transgressiv“ oder standen gar im Gegensatz zu nationaler Staatlichkeit. Imperiale Biographien oder jene von Diplomaten zeugen beispielsweise von einem zum Teil lebhaften staatlichen Interesse an Formen der (kontrollierten) Grenzüberschreitung. 16

Viele der bereits angesprochenen Elitemigranten lassen sich dagegen einem zweiten Typ transnationaler Leben zuordnen, der Figur des ,transnationalen Mittlers“. Auch den Biographien von jenen, die zwischen Gesellschaften vermittelten, globale Brücken bauten oder grenzüberschreitende Netzwerke etablierten, hat die transnationale Geschichte besondere Aufmerksamkeit geschenkt. Forschungen zum atlantischen Raum in der Frühen Neuzeit (als kulturelle Grenzen noch weit wichtiger waren als staatliche) arbeiten mit der Figur des cultural broker, also Dolmetscher, Missionare und Forscher, die als Kulturvermittler z.B. zwischen europäischen und indigenen Kulturen fungierten (vgl. Häberlein/Keese 2010). Für die transatlantische Geschichte der letzten Nachkriegszeit dagegen haben Arndt Bauerkämper, Konrad Jarausch und Marcus Payk den Begriff des „transatlantischen Mittlers“ eingeführt, welcher als rückkehrender Emigrant oder amerikanischer Europaexperte eine ähnliche Brückenfunktion einnahm und bei grenzüberschreitenden Kultur- und Institutionentransfers im Zuge der „Westernisierung“ Deutschlands eine wichtige Rolle spielte (Bauerkämper/Jarausch/Payk 2005).

Die klischeehafte Metapher des „Brückenbauers“ ist dabei irreführend und zu statisch, um die dynamische Qualität von transnationalen und transkulturellen Austauschprozessen zu erfassen. Transfers wirkten auf alle beteiligten Gesellschaften, und die Rolle des Vermittlers ist eher die eines aktiven Übersetzers als jene eines passiven Transmitters. In diesem Zusammenhang sei schließlich auf den von Doris Bachmann-Medick und anderen propagierten translational turn verwiesen und die Erkenntnis, dass sich bei transnationalen Übersetzungsprozessen alle involvierten Personen, Ideen, und Gesellschaften gleichermaßen wandeln (Bachmann-Medick 2009). Im Gegensatz zur Vorstellung von eher linearen zwischengesellschaftlichen Akkulturationsprozessen zwischen Sende- und Empfängergesellschaft der frühen Kulturtransferforschung bietet die Perspektive der kulturellen Übersetzung eine Betonung von aktiver Aneignung und Reziprozität (Lässig 2012). Gerade Elitemigranten mit ihren transnationalen Leben spielten dabei häufig die vermittelnde Rolle von Übersetzern, die aus einer Position des „Dazwischen“ Neues schufen (Shaw 2014). Solche kreativen „transatlantischen Mittler“" standen im Zentrum der Forschung zum Transatlantic Perspectives-Projekt.

15 Zur Frage der Grenzüberschreitung vgl. Lüthi/Rürup (2010).

16 Als Beispiel zur Rolle solcher Akteure bei der Generierung von colonial knowledge siehe Habermas, (2010). 


\section{Transatlantic Perspectives: Transatlantische Karrieren als Transnationale Geschichte}

In der klassischen Periodisierung der Geschichte globaler Austauschprozesse gilt die Mitte des zwanzigsten Jahrhunderts als eine Zeit der Deglobalisierung. Weltkriege, Wirtschaftskrise und Kalter Krieg trugen zu einer Desintegration transnationaler Prozesse und zur regionalen Blockbildung bei, die einen Bruch bildeten zwischen der ersten Welle moderner „Globalisierung“ bis 1914 und einer zweiten Welle ab den 1970er Jahren. ${ }^{17}$ Im transatlantischen Binnenverhältnis gab es im selben Zeitraum eine signifikante wirtschaftliche und politische Machtverschiebung zugunsten der USA, sodass aus vormals wechselseitigen transatlantischen Transfers zunehmend eine einseitige „Amerikanisierung“ Europas geworden zu sein schien. Dabei waren die Gesellschaften beiderseits des Atlantiks jedoch besonders in den Nachkriegsjahrzehnten institutionell, transporttechnisch und medial enger verbunden als je zuvor. Der Blick auf die transnationalen Leben von zahlreichen Elitemigranten, die sich zwischen den 1920er und 1960er Jahren im atlantischen Raum bewegten, zeigt dann auch, dass sowohl die Annahme einer „Deglobalisierung“ als auch die einer zunehmend einseitigen „Amerikanisierung“ deutlich qualifiziert werden müssen. Vielmehr ergibt sich ein Bild der longue durée gelebter Globalität und transnationaler Austauschprozesse auch (und zum Teil gerade) in Zeiten von Krieg und Krise.

Ziel der Nachwuchsforschergruppe Transatlantic Perspectives war es, anhand von Migrantenkarrieren transatlantische Austauschprozesse zwischen Europa und den USA um die Mitte des zwanzigsten Jahrhunderts in zentralen Gesellschaftsbereichen der westlichen Hochmoderne freizulegen. Dabei befassten sich vier einzelne Untersuchungen mit der Rolle von Migranten in jeweils verschiedenen Professionen und Gesellschaftsbereichen wie der Stadtforschung, der angewandten Sozialforschung, in multinationalen Unternehmen und im Bereich des Consumer Engineering, d.h. von Produktgestaltung und Markt- und Konsumforschung. ${ }^{18}$ Verschiedene Workshops haben nach dem Selbstverständnis und der Selbstverortung der Migranten zwischen Europa und den USA gefragt, nach ihrem Beitrag zur transatlantischen Vernetzung und nach ihrer Funktion als zwischengesellschaftliche Übersetzer in der Nachkriegsmoderne (vgl. z.B. Logemann/Gabaccia/Kohlstedt 2014). Auf der Projektwebseite www.transatlanticperspectives.org wurden darüber hinaus mehr als sechzig kurze biographische Skizzen von europäischen Migranten mit Karrieren in den unterschiedlichsten Feldern von Politik und Staatswissenschaft bis hin zu Populärkultur und Film gesammelt, die von knapp dreißig externen Autorinnen und Autoren beigesteuert wurden.

Die Verknüpfung von transatlantischen Karrieren mit transnationalen Netzwerken und Transfers stand dabei jeweils im Vordergrund der Darstellung. Es handelt sich hierbei jedoch nicht um Biographien im klassischen Sinne oder um das Betrachten von „transnationalen Leben“" in ihrer Totalität. Zwar haben wir zum Teil auch nach persönlichen Erfahrungen, etwa im Wandel der Selbstwahrnehmung und in der Auseinandersetzung mit Europa als Idee und Identifikationsgröße gefragt. Unser Hauptinteresse galt aber dem beruflichen Werdegang, also den „transnationalen Karrieren“

$17 \mathrm{Zu}$ globalgeschichtlichen Periodisierungen vgl. Osterhammel/Petersson ${ }^{5}$ 2012: 7-27.

18 Kurze Skizzen der Einzelprojekte von Andreas Joch, Jan Logemann, Barbara Louis und Corinna Ludwig finden sich in The German Historical Institute at 25 Years, GHI Bulletin Supplement 8 (2012). 
der Migranten und den Transfer- und Austauschprozessen, die diese enthielten. Welche Auswirkungen hatten die „Grenzgänge“ der Migrationserfahrung auf das professionelle Wirken der Migranten, und welche Auswirkungen hatten die „Grenzgänger“ selber auf ihr professionelles Umfeld? Welche Einflüsse und Übersetzungen durch die „transatlantischen Mittler“ lassen sich erkennen, die beispielsweise die amerikanische Welt der Waren oder die Ausformung amerikanischer Metropolen der 1940er und 1950er Jahre in einem neuen, transnationalen Licht erscheinen lassen?

Dabei wurde sehr rasch die Kontinuität wechselseitiger transatlantischer Transfers über die üblichen Zäsuren der Jahrhundertmitte hinweg deutlich. So sticht die Bedeutung der intellektuellen Emigration der 1930er Jahre mit ihren Transfers und Übersetzungsleistungen von Europa in die USA hervor. Mehr als 130.000 oft gut ausgebildete Flüchtlinge und Exilanten kamen allein aus dem deutschsprachigen Raum, von denen viele zur Kategorie der „Elitemigranten“ gerechnet werden können (vgl. Unger 2009). Nicht allen gelang eine erfolgreiche Fortsetzung ihrer Karrieren, doch unzählige Emigranten konnten sich in einflussreichen Positionen der amerikanischen Wirtschaft, Verwaltung, Wissenschaft und Kulturszene etablieren. In Folge der Krise und im Kontext des Weltkrieges gab es sogar eine verstärkte Nachfrage nach ,europäischer" Expertise in Staat, Wirtschaft und Militär der USA. Der American Way of Life und die amerikanische Demokratie der Jahrhundertmitte wurden so durch die transnationalen Leben unzähliger Immigranten und Emigranten entscheidend mitgeprägt. 19

Statt eines einseitigen Transfers als Brain Drain handelte es sich vielmehr um einen reziproken Wissensaustausch im Sinne einer Brain Circulation. Nach dem Zweiten Weltkrieg waren es nämlich oft dieselben Migranten die jene, nun als „Amerikanisierung" wahrgenommene, kulturelle Übersetzung in die andere Richtung leisteten (vgl. Bauerkämper/Jarausch/Payk 2005). Ob im Rahmen der Remigration oder über verschiedene institutionelle Netzwerke, die Rekonstruktion Nachkriegseuropas, die „Westernisierung“ politischer Kulturen oder die „Modernisierung“ von Wirtschaft, Städten, Kultur und Gesellschaft war beeinflusst von den transnationalen Leben zahlreicher ,transatlantischer Mittler.“ Das, was transferiert wurde, waren dabei vielfach modifizierte „Re-importe“ ohnehin transatlantisch generierter Wissensbestände und Kulturtechniken, wie ich im folgenden Abschnitt am Beispiel der Konsumforschung kurz illustrieren werde. Trotz der globalen Bedeutung des amerikanischen Demokratiemodells im Kalten Krieg oder der Strahlkraft der US-Konsumgesellschaft war der Atlantik also auch nach 1945 keine Einbahnstraße. Jene multilateralen Transferprozesse, die Daniel Rodgers so eindrucksvoll für die erste Hälfte des zwanzigsten Jahrhunderts beschrieben hat, setzten sich in vielen Gesellschaftsbereichen durchaus fort. Aus der Perspektive der zahlreichen transnationalen Leben, die wir in diesem Projekt untersucht haben, erscheint das von Henry Luce proklamierte American Century eher als ein vom wechselseitigen Austausch charakterisiertes Transatlantic Century (Nolan 2012.

Den „europäischen“ Stimmen in diesem transatlantischen Diskurs haben wir dabei besondere Aufmerksamkeit geschenkt. So sammelt der Band More Atlantic Crossings? biographische Einzelstudien zu europäischen Migranten und prominenten transatlantischen Karrieren von Mittlern, Netzwerkern, aber auch von Grenzgängern

19 Greenberg (2014) postulierte mit Blick auf den Einfluss der Emigranten jüngst sogar ein „Weimar Century“. Für den Konsumbereich vgl. Logemann (2013). 
(Logemann/Nolan 2014). Politische Denker wie Hans Speier halfen, dem entstehenden National Security State der USA Form zu geben (Bessner 2014). Akademiker wie die Historiker Peter Gay und George Mosse oder der Sozialwissenschaftler Franz Neumann spielten die Rolle von methodischen und konzeptionellen Mittlern in den Wissenschaften und von kulturellen Übersetzern zwischen Europa und den USA für eine breitere Öffentlichkeit (Wheatland/Neumann 2014). Sie waren beteiligt an der Konstruktion von transnationalen diskursiven Räumen wie jener ,atlantischen Gemeinschaft", die durch grenzüberschreitende Netzwerke von gesellschaftlichen Eliten und Diplomaten getragen wurden. Andere Formen transnationaler Leben forderten diese transatlantische Raumordnung dagegen eher heraus. „Transgressive“ kosmopolitische Lebensläufe wie jene von Aurelio Peccei und Alexander King, den Begründern des Club of Rome, versuchten die Ost-West Trennung des Kalten Krieges zu durchbrechen und eröffneten in den 1960er Jahren eine globalere Perspektive, die über den atlantischen Raum hinauswies (Albrecht 2014). Auf eine ganz andere Art bediente auch der Ökonom Wilhelm Röpcke eine globalere Perspektive und sprach zu einer transnationalen Gegenöffentlichkeit konservativer Kritiker keynesianischer Wachstums- und Entwicklungspolitik (Slobodian 2014). In der Analyse solcher transatlantischer Leben findet sich eine Kontinuität von grenzüberschreitendem Austausch über Gesellschaftsentwicklung, die von den wechselseitigen Verständigungen über Entwürfe der Moderne in der Zwischenkriegszeit bis hin zu einer globalen Zivilgesellschaft der späten 1960er und frühen 1970er Jahre reicht.

\section{Die Wirkungsmacht transnationaler Leben: Biographische Perspektiven auf transatlantische Prozesse}

Welche konkrete Bedeutung haben nun transnationale Leben für solch eine Kontinuität transnationaler Entwicklungen und was genau zeigt uns der biographische Ansatz? Anhand von kurzen Beispielen aus meiner Forschung zu Transferprozessen im Bereich des Marketings und Consumer Engineering möchte ich im Folgenden drei Aspekte der transnationalen Wirkungsmacht von Migrantenkarrieren kurz skizzieren. ${ }^{20}$ Zunächst soll die Bedeutung von transnationalen Karrieren für grenzüberschreitende Wissenstransfers und deren Reziprozität herausgestellt werden. Dann werde ich auf den Aspekt der Nachhaltigkeit transatlantischen Austauschs aufgrund von grenzüberschreitender Netzwerkbildung eingehen. Schließlich soll die Rolle von Migranten als kreative Übersetzer in Transferprozessen beleuchtet werden.

\section{Grenzüberschreitender und reziproker Wissenstransfer durch Migration}

In der Folge der Transportrevolution des neunzehnten Jahrhunderts und einer immer weiter zunehmenden medialen Vernetzung bedurfte es für den grenzüberschreitenden Austausch von Wissen oder Waren eigentlich nicht zwingend der Migranten. Eine Welt jenseits der Grenzen konnte in der Neuzeit durch Bücher oder Briefe, Kolonialwaren oder Fernsehbilder auch lokal von jenen erfahren werden, die gar nicht oder nur sehr begrenzt mobil waren. Dennoch waren transnationale Mittler in gesellschaftlichen Funktionseliten oft von entscheidender Bedeutung für Transfers und vor allem

20 Die folgenden Beispiele stammen aus meinem laufenden Habilitationsprojekt zu Transfers in Marketing und Produktdesign durch europäische Emigranten (vgl. Logemann 2013). 
für das Entstehen kreativer Innovationen im Transferprozess. Das Beispiel des Eingangs eingeführten Paul Lazarsfeld kann dies illustrieren, da seine Karriere den wechselseitigen Austausch in der empirischen Markt- und Sozialforschung nachhaltig beeinflusst hat. 21

Paul Lazarsfeld hatte im Wien der Zwischenkriegszeit eine wirtschaftspsychologische Forschungsstelle aufgebaut, die (inspiriert zum Teil von amerikanischen Studien) empirisch-methodisch und sozialpsychologisch innovative Forschung betrieb. Bekannt wurde vor allem eine Studie zu den Arbeitslosen von Marienthal, aber der finanzielle und methodische Kern des Instituts bestand aus Marktforschungsstudien für Unternehmen aus Österreich und anderen europäischen Ländern. Als Lazarsfeld Mitte der 1930er Jahre mit Hilfe der Rockefeller Stiftung in die USA übersiedelte, lag ein Schlüssel für seinen anfänglichen Erfolg in diesem Interesse an Konsumforschung. Der Wiener Soziologe war zunächst in die Vereinigten Staaten gekommen, um amerikanische Methoden der Markt- und Sozialforschung eingehender zu studieren, aber er stellte bald fest, dass die Amerikaner ebenso an seiner Expertise interessiert waren. Versiert in den neuesten Erkenntnissen der europäischen Individual- und Sozialpsychologie und methodisch reflektiert in seinem Zugang zur Verbrauchermotivation, war Lazarsfeld für die Marketingabteilungen krisengebeutelter amerikanischer Unternehmen gleichermaßen interessant wie für den zunehmend verbraucherorientierten Staat des New Deal.

Lazarsfeld begann in den 1930er Jahren, ein transnationales Leben zwischen amerikanischen Institutionen, Emigrantenzirkeln und Kontakten zurück nach Europa zu führen. Seine zunächst noch auf Wiener Forschung basierenden methodischen Überlegungen bereicherten die amerikanische Konsum- und Medienforschung der 1930er Jahre, wobei sich seine Ansätze zur Konsumentenpsychologie im amerikanischen Kontext weiterentwickelten. Lazarsfeld baute nach dem Vorbild seiner Wiener Forschungsstelle eine Reihe von Forschungsprojekten und Instituten in den USA auf; das bekannte Radio Research Project etwa war mit der Princeton University verknüpft und wurde von der Rockefeller Stiftung und dem Rundfunksender CBS finanziell mit unterstützt. Ab 1940 war Lazarsfeld dann an der Columbia University, wo er das Bureau of Applied Social Research (BASR) aufbaute. Medienwirksamkeitsstudien, Panelstudien von Konsumenten, Fokusgruppen in der Werbeforschung ebenso wie die psychologische Motivforschung lassen sich in Ansätzen auf das institutionelle und persönliche Umfeld Lazarsfelds zurückführen. Die psychologisierte Welt der Waren und die tiefenpsychologische Vermessung der Konsumentenseele im Nachkriegsamerika fußten somit auf einem regen transatlantischen Austausch, bei dem Paul Lazarsfeld eine zentrale Rolle spielte und der sich in den Nachkriegsjahrzenten im Sinne einer reziproken Brain Circulation fortsetzen sollte. Seine physische Präsenz als Lehrer und Forscher war für das Zustandekommen transnationaler Transfers zentral und seine Adaption an die jeweiligen lokalen Kontexte trug entscheidend zum innovativen Charakter seines Wirkens bei.

$21 \mathrm{Zu}$ Lazarsfelds Biographie siehe u.a. Langenbucher (2008). Zu seiner Rolle in transatlantischen Transfers siehe auch Fleck (2007). 


\section{Individuelle Netzwerke und die Verstetigung von Transferprozessen}

Dass Lazarsfelds methodische Interventionen so erfolgreich waren, lag auch daran, dass er ein weites persönliches Netzwerk in der Emigration aufbaute und sich selber erfolgreich in bestehende transnationale Netzwerke integrierte. Die Bedeutung von Netzwerken für den grenzüberschreitenden Transfer ist in der Forschung jüngst verstärkt untersucht worden - zumal der von Manuel Castells geprägte Begriff der „Netzwerkgesellschaft“ zu einem Leitbegriff der Globalisierungsforschung geworden ist. Das Konzept des Netzwerks bleibt gelegentlich unscharf, jedoch geht es im Kern um gesellschaftliche Organisationsformen zwischen der Offenheit des Marktes und der Geschlossenheit hierarchischer Institutionen, die auf überlappenden persönlichen Beziehungen und auf Vertrauensverhältnissen basieren. An den Knotenpunkten dieser Netzwerke stehen Organisationen und Personen, die einen dezentralen und transnationalen Austausch von Wissen (oder auch Macht und Kapital) befördern (Mittag/Unfried 2008). Die soziale Netzwerkanalyse hatte ihren Anfang u.a. mit solchen emigrierten Sozialforschern wie Kurt Lewin und dessen Arbeiten zum sozialen Feld oder eben mit Paul Lazarsfeld und dessen Beschäftigung mit persönlichen Netzwerken und der kommunikativen Wirkungsmacht sozialer Multiplikatoren (Neurath/Krempel 2008). Netzwerke, so die Erkenntnis, bestimmen die Handlungsräume von Individuen und sind zentral für die kommunikative Strukturierung von Transfers. Im Freilegen dieser Netzwerke liegt dann auch ein Beitrag eines akteursfokussierten, biographischen Zugriffs auf transnationale Geschichte.

Paul Lazarsfelds transatlantische Karriere war geprägt durch seine Fähigkeit, Netzwerke aufzubauen, zu unterhalten und zu nutzen. Zum einen war er Teil institutioneller Netzwerke, etwa durch seine Beziehung zur Rockefeller Stiftung, die sich von der Zwischenkriegs- bis in die Nachkriegszeit durchzog, oder durch diverse Verbindungen zu akademischen Einrichtungen, an denen er beschäftigt war, sowie durch die Institute, die er in Europa und den USA aufbaute. Lazarsfelds Netzwerke erstreckten sich bald auch auf staatliche Stellen, die er bei der Konsumforschung im New Deal, bei der psychologischen Kriegsführung an der Heimatfront oder bei internationalen Kommunikationsstrategien im Kalten Krieg beriet. Ebenso hatte er gleich nach seiner Ankunft Kontakte zu amerikanischen Firmen geknüpft, denen er bis weit in die Nachkriegszeit in Marktforschungsfragen beratend zur Seite stand. Schließlich hatte er ein weitgreifendes persönliches Netzwerk aufgebaut, zu dem zahlreiche andere Emigranten in den Sozialwissenschaften gehörten. Sein ehemaliger Wiener und späterer New Yorker Kollege Hans Zeisel sprach so von einer „Wiener Schule der Marktforschung“ in den USA, der u.a. auch Ernst Dichter und Herta Herzog mit angehörten und die in ihrer Gesamtheit die angewandte Sozialforschung im transatlantischen Raum signifikant beeinflusst hat (Zeisel 1988). Solche Verstärkereffekte für den Transfer durch Netzwerke und die Rolle bestimmter Elitenmigranten als Multiplikatoren, wie sie hier am Beispiel Lazarsfelds und der empirischen Sozialforschung vorgestellt wurden, lassen sich für viele Bereiche nachzeichnen. In dem für den Konsum ebenfalls zentralen Bereich von Design, Architektur und Gestaltung sei etwa auf das Netzwerk emigrierter Bauhaus-Künstler um Walter Gropius hingewiesen.22 Auch hier wurde der Transfer von Stilinnovationen und Designkonzepten durch persönliche und institutio-

22 Zur transatlantischen Geschichte des Bauhauses vgl. u.a. James (2006). 
nelle Netzwerke befördert, die von Europa in die USA und zurück nach Europa reichten.

Die reziproke Dimension und die Bedeutung des Rücktransfers in Netzwerken treten beim Studium transnationaler Leben besonders deutlich hervor. Wie die Bauhausdesigner wirkten auch Lazarsfeld und sein Umfeld auf Europa zurück, und wie so viele Migranten kehrte er wenigstens zeitweilig als reisender Sojourner in seine alte Heimat zurück. 1963 initiierte Lazarsfeld mit dem ebenfalls aus Wien emigrierten Ökonomen Oskar Morgenstern das Institut für Höhere Studien in seiner Heimatstadt und konnte dabei auf seine vielfältigen institutionellen und persönlichen Netzwerke zurückgreifen. Finanziert mit Hilfe der Ford Stiftung, bot das Institut nicht nur ehemaligen Emigranten wie Charlotte Bühler und Friedrich Hayek die Möglichkeit, durch Gastaufenthalte nach Europa zurückzuwirken, sondern es sollte auch bei der von James Coleman angesprochenen Verbreitung der ,amerikanischen“ Sozialforschung in Europa helfen. ${ }^{23}$ Die Multidirektionalität von Transferprozessen in Netzwerken wird durch die langfristige biographische Perspektive besonders greifbar.

\section{Transfers als Übersetzung: die aktive Leistung der Mittler}

Der akteurszentrierte, biographische Blick auf transnationale Transferprozesse unterstreicht schließlich die Bedeutung von aktiven Übersetzungsleistungen. Simone Lässig schrieb jüngst zur Funktion der Übersetzung im Transfer: „Es geht nicht mehr um bloßen Transfer von Kultur, sondern um Transformationen; es geht nicht mehr um ,Übernahme', sondern um komplexe Prozesse des Aushandelns und Aneignens von Ideen oder Praktiken und des Herstellens von Bedeutung; es geht [...] nicht zuletzt auch um jene Akteure, die die Verständigung über Kultur in unterschiedlichen Räumen und Zeiten getragen und maßgeblich beeinflusst haben“ (Lässig 2012: 95). Solche Formen der Übersetzungen in verschiedene Richtungen lassen sich am Beispiel Lazarsfelds und seines Netzwerkes ebenfalls gut illustrieren.

Schon kurz nach seiner Ankunft in den Vereinigten Staaten begann Paul Lazarsfeld, sich auf verschiedene Weise als transatlantischer „Übersetzer“ zu positionieren. Auf einer sehr direkten Ebene übersetzte er seine Wiener Forschungen zu Konsumentenmotivationen ins Englische, um diese in amerikanischen Publikationen zu veröffentlichen. Dies war jedoch nur ein erster Schritt, denn er ,übersetzte“ auf einer anderen Ebene auch seine Forschungsdesigns in Marktstudien für amerikanische Firmen, wobei er diese dem amerikanischen Kontext neu anpassen musste. Ebenso war er daran beteiligt, den theoretischen Hintergrund seiner Forschung seinen neuen amerikanischen Kollegen zu vermitteln, indem er etwa die sozialpsychologischen Ansätze Kurt Lewins der dortigen Expertengemeinde für Sozialforschung nahebrachte. Schließlich bot er sich auch als Übersetzer europäischer Märkte an, als „Mann in Europa“ (wohin er zunächst noch häufig zurückreiste), der über die Psychological Corporation und über seine Kontakte in Wien Marktstudien für amerikanische Firmen auf dem Kontinent vermitteln konnte. Für das Publikum in Österreich schrieb er dagegen Berichte über die amerikanische Konsumforschung und Konsumpolitik im New Deal. So vermittelte Lazarsfeld aktiv als Übersetzer zwischen zwei Gesellschaften und beförderte wissenschaftliche wie wirtschaftliche Transfers.

23 Coleman hatte dort ebenfalls einen Gastaufenthalt (vgl. Fleck 2000). 
Der Motivforscher Ernst Dichter, der ebenfalls der „Wiener Schule“ der Marktforschung entstammte, unternahm in den Nachkriegsjahrzehnten sehr ähnliche Übersetzungsanstrengungen. 24 Für die Europäer übersetzte er moderne, ,amerikanische“ Ansätze der Marktforschung (vornehmlich die von ihm mitentwickelte qualitative und tiefenpsychologisch arbeitende Motivforschung), indem er sich auf Vorträgen, auf Kongressen und in Publikationen an die europäischen Marktforscher richtete und in Marktstudien für europäische Firmen diese Konzepte praktisch anwandte. Amerikanischen Unternehmen hingegen bot Dichter in seinen Studien und in Essays für Fachzeitschriften seine Expertise an, um auf dem europäischen Markt zu reüssieren. Er übersetzte europäische Konsumgewohnheiten, Verbrauchererwartungen oder die Unterschiede im Geschäftsgebaren. Dichter war sich seiner Rolle als transatlantischer Mittler sehr bewusst und reflektierte offen darüber, wie vorteilhaft es für ihn war, dass er - obwohl mittlerweile Amerikaner - von Europäern als einer der Ihren erkannt wurde: "[Europeans] accept me and probably many others; in my particular case because I can combine European answers with American know-how. [...] I do speak their languages and they will sort of hug me and say, 'Well, you're really one of us." 25

Dichter macht hier die kreative Übersetzungsleistung von Elitemigranten explizit - er half durch Adaption und Translation das zu Transferierende zu „lokalisieren“ und den spezifischen Gegebenheiten anzupassen. Zugleich nimmt er als Emigrant dem Transfer ein wenig die Qualität des Fremden (etwa im negativen Sinne einer „Amerikanisierung“), sondern erlaubt es, das Neue und „Moderne“ aus den USA in eine (konstruierte) Tradition einzubetten, die in diesem Fall zur europäischen Psychologie und Sozialforschung der Zwischenkriegszeit reicht. Dichters Zitat verweist schließlich auch darauf, dass die transnationalen Leben und transatlantischen Karrieren von vielen der Individuen, die wir im Rahmen des Transatlantic Perspectives-Projekt untersucht haben, von ihnen selber positiv in eine Stärke gewandt wurden, die sich in geschäftlichen und professionellen Zusammenhängen strategisch einsetzen ließ.

\section{Brüche, Scheitern und ,blinde Flecken“ in der Beschäftigung mit Transnationalen Leben von Elitemigranten}

Die transnationalen Leben von Paul Lazarsfeld oder Ernest Dichter lassen sich sehr einfach als Erfolgsgeschichten erzählen, und darin verbirgt sich auch eine gewisse Problematik des Blicks auf Elitenmigranten als Akteure transnationaler Geschichte. Ich möchte abschließend kurz auf blinde Flecken in der Forschungsperspektive unseres Projekts verweisen, das sich ja explizit mit den Lebensentwürfen und Karrieren von gesellschaftlichen Eliten auseinander gesetzt hat, also jenen, die unternehmerisch, sozial, akademisch oder politisch erfolgreich wirken konnten und eben dadurch zu grenzüberschreitenden Austauschprozessen beigetragen haben. Dieser Fokus bedeutet jedoch auch, dass Brüche, Verlust oder gar Scheitern eher selten im Mittelpunkt der Überlegungen standen.

Dabei sind gerade solche Erfahrungen beim Grenzenüberschreiten häufig zentral für die Selbstwahrnehmung ihrer transnationalen Leben durch Migranten. Die Auto-

$24 \mathrm{Zu}$ Dichters transatlantischer Biographie vgl. u.a. Gries/Schwarzkopf (2007).

25 Ernst Dichter, "Business Abroad Article - (Rough Copy)," 28. März 1967, Nachlass Ernest Dichter, Hagley Museum and Library, Box 169. 
biographien von Emigrantinnen und Exilanten, die zur Migration gezwungen wurden, sind geprägt von der Auseinandersetzung mit Brüchen in der eigenen Lebensgeschichte und von persönlichen Verlusterfahrungen (Depkat 2005). Karriereverläufe ließen sich in Folge von Migration oft nicht einfach fortsetzen oder mussten in ihrem Verlauf stark modifiziert werden - die vergleichsweise glatten transatlantischen Karrieren von Lazarsfeld oder Dichter sind in diesem Sinne eher Ausnahmen. ${ }^{26}$ Dabei ließen sich gerade aus dem Scheitern von Karrieren und Transfers, dem Zerfallen von Netzwerken oder dem Misslingen von Übersetzungen ebenfalls wichtige Einsichten in die Strukturen transnationaler Geschichte gewinnen.27

Die explizite Suche nach „transatlantischen Mittlern“ und jenen, die Transfer- und Austauschprozesse vorangetrieben haben, darf nicht den Blick auf andere Formen transnationaler Leben verstellen. Der oben diskutierte Typ der „transgressiven Biographie" lässt sich auch unter Elitemigranten finden, jedoch sind es weniger die kulturvermittelnden oder vernetzenden Qualitäten transnationaler Leben, die sich dann zeigen. Vielmehr stellen diese Biographien eine Herausforderung dar, die die Grenzen und Konflikte transnationaler Prozesse klarer herausstellt, als es der Fokus auf die Mittler leisten kann. Marginale Gruppen von Migranten und jene vernacular cosmopolitans, die aus einer minoritären Rolle grenzübergreifend kulturverändernd wirken, können hier eine ganz ähnliche Rolle spielen. ${ }^{28}$ Die marginalen Grenzgänger und jene, die scheitern, sind quellentechnisch schwieriger zu fassen als Elitemigranten mit Nachrufen und wohl-archivierten Nachlässen, jedoch sind ihre transnationalen Biographien gut geeignet, um allzu rosige Visionen einer globalisierten und transnationalen Welt zu hinterfragen.

Biographik ermöglicht so auch einen Blick auf die Kosten von Transnationalität und von Prozessen globalen Austauschs im zwanzigsten Jahrhundert, die in der Perspektive auf hybride Kreationen, kosmopolitische Lebensentwürfe und befreiende Grenzüberschreitungen mitunter verloren gehen. Wenigen gelingt es so problemlos und mit so viel Erfolg, zwischen den Welten zu wechseln und dabei ganze Forschungszweige und Professionen transnational zu befruchten wie Paul Lazarsfeld, mit dessen Nachruf ich diese Überlegungen begonnen habe. Lazarsfeld war frühes Mitglied einer Elite, die der Sozialtheoretiker Craig Calhoun mit Blick auf das frühe 21. Jahrhundert als die kosmopolitische Klasse der Frequent Travellers bezeichnet hat (Calhoun 2009). Wie bei Bhabhas global cosmopolitans zeichnen sich deren transnationale Leben in vieler Hinsicht durch eine große Nähe zu den Zentren gesellschaftlicher Macht aus. Viele der ,transatlantischen Mittler“" mit ihren Netzwerken und Übersetzungsleistungen waren aktive Konstrukteure jener globalen Machtstrukturen, die die Globalisierung heute für manche eher als Dystopie denn als Utopie erscheinen lassen. Bei aller Faszination für die innovativen Leistungen wechselseitigen, grenzüberschreitenden Austausches, so hat Ian Tyrell gewarnt, darf die transnationale Geschichte auch Fragen von Macht und Machtungleichgewichten nicht aus den Augen verlieren (Tyrell 2009); für eine transnationale Biographik gilt dies ebenso.

26 Zur Frage von Brüchen und Möglichkeiten in Emigrantenkarrieren in den Sozialwissenschaften vgl. auch Louis (2013).

27 Für Überlegungen zur Geschichte des Scheiterns für den ähnlich auf Erfolgsgeschichten orientierten Bereich der Unternehmensgeschichte siehe Köhler/Rossfeld (2012).

28 So auch die Aufforderung, marginalisierte Individuen stärker in der transnationalen Biographik zu berücksichtigen, bei Harders (2014). 


\section{LITERATUR}

Albrecht, Christian (2014): The Atlantic Community in a Global Context: Global Crisis and Atlanticism within the Context of the Club of Rome, in: Jan Logemann und Mary Nolan (Hg.) (2014): More Atlantic Crossings? European Voices and the Postwar Atlantic Community, (GHI Bulletin Supplement 10), Washington, 169-182.

Bachmann-Medick, Doris (2009): The Translational Turn, in: Translation Studies 2, 2-16.

Bauerkämper, Arndt, Konrad Jarausch, Marcus M. Payk (Hg.) (2005): Demokratiewunder. Transatlantische Mittler und die kulturelle Öffnung Westdeutschlands 1945-1970, Göttingen.

Bender, Thomas (2007): A Nation Among Nations: America's Place in World History, New York.

Berghahn, Volker (2004): Transatlantische Kulturkriege: Shepard Stone, die Ford-Stiftung und der europäische Antiamerikanismus, Stuttgart.

Berghahn, Volker und Simone Lässig (2008): Biography between Structure and Agency: Central European Lives in International Historiography, New York.

Berghoff, Hartmut und Uwe Spiekermann (2010): Immigrant Entrepreneurship: GermanAmerican Business Biography 1720 to the Present, in: GHI Bulletin 47, 69-82

Berghoff, Hartmut und Andreas Fahrmeir (2013): Unternehmer und Migration. Einleitung [zu einem Themenheft], in: Zeitschrift für Unternehmensgeschichte 58, 141-148.

Bessner, Daniel (2014): Weimar Social Science in Cold War America: The Case of the Political-Military Game, in: Jan Logemann und Mary Nolan (Hg.) (2014): More Atlantic Crossings? European Voices and the Postwar Atlantic Community, (GHI Bulletin Supplement 10), Washington, 91-109.

Bhabha, Homi (1994): Locations of Culture, London.

Bhabha, Homi (2004): Looking Back, Moving Forward: Notes on Vernacular Cosmopolitanism, Preface to Routledge Classics Edition of The Location of Culture, London, ix-xxv.

Brinkmann, Tobias (2010): Taking the Global View: Reconsidering Migration History after 1800, in: Neue Politische Literatur 55/2, 213-232.

Calhoun, Craig (2009): The Class Consciousness of Frequent Travelers: Toward a Critique of Actually Existing Cosmopolitanism, in: Saurabh Dube (Hg.), Enchantments of Modernity: Empire, Nation, Globalization, Abingdon, 310-340.

Coleman, James (1976): Obituary Paul F. Lazarsfeld, in: ASA Footnotes, Dec. 1976, 7.

Conrad, Sebastian und Jürgen Osterhammel (Hg.) (2009): Das Kaiserreich transnational: Deutschland in der Welt 1871-1914, Göttingen.

Dahlmann, Dittmar und Reinhold Reith (Hg.) (2008): Elitenwanderung und Wissenstransfer im 19. und 20. Jahrhundert, Essen.

Deacon, Desley, Penny Russell und Angela Woollacott (Hg.) (2011): Transnational Lives: Biographies of Global Modernity, 1700-Present, Basingstoke.

Depkat, Volker (2005): Der biographische Ort des Exils, in: Autobiografie und wissenschaftliche Biografik. Jahrbuch Exilforschung 23, 30-56.

Depkat, Volker (2014): The Challenges of Biography: European-American Reflections, in: GHI Bulletin 55, 39-48

Dichter, Ernst (1967): "Business Abroad Article - (Rough Copy)", 28. März 1967, Nachlass Ernest Dichter, Hagley Museum and Library, Box 169.

Domhardt, Konstanze (2011): The Heart of the City: CIAM-Debatten zwischen Europa und Nordamerika 1933-1951, Zürich.

Etzemüller, Thomas (2012): Biographien. Lesen - erforschen - erzählen, Frankfurt am Main.

Fechter, Anne-Meike und Katie Walsh: Examining 'Expatriate' Continuities: Postcolonial Approaches to Mobile Professionals, in: Journal of Ethnic and Migration Studies 36, 1197-1210. 
Fleck, Christian (2000): Wie Neues nicht entsteht: Die Gründung des Instituts für Höhere Studien in Wien durch Ex-Österreicher und die Ford Foundation, in: Österreichische Zeitschrift für Geschichtswissenschaften 11, 129-178.

Fleck, Christian (2007): Transatlantische Bereicherungen. Zur Erfindung der empirischen Sozialforschung, Frankfurt am Main.

Gabaccia, Donna (2012): Foreign Relations. American Immigration in Global Perspective, Princeton.

Gemelli, Giuliana und Roy MacLeod (Hg.) (2003): American Foundations in Europe: GrantGiving Policies, Cultural Diplomacy, and Trans-Atlantic Relations, 1920-1980, Brüssel.

Grazia, Victoria de (2005): Irresistible Empire: America's Advance through Twentieth Century Europe, Cambridge.

Green, Nancy (2014): The Other Americans in Paris. Businessmen, Countesses, Wayward Youth, 1880-1941, Chicago.

Greenberg, Udi (2014): The Weimar Century: German émigrés and the Ideological Foundations of the Cold War, Princeton.

Gries, Rainer und Stefan Schwarzkopf (2007): Ernest Dichter: Doyen der Verführer, Wien.

Guterl, Matthew Pratt (2013): The Futures of Transnational History: Comment to AHR Forum Transnational Lives in the Twentieth Century, in: American Historical Review 118, 130139.

Häberlein, Mark und Alexander Keese (Hg.) (2010): Sprachgrenzen - Sprachkontakte - kulturelle Vermittler. Kommunikation zwischen Europäern und Außereuropäern (16.-20. Jahrhundert), Wiesbaden.

Habermas, Rebekka (2010): Wissenstransfer und Mission: Sklavenhändler, Missionare und Religionswissenschaftler, in: Geschichte und Gesellschaft 36, 257-284.

Habermas, Rebekka und Sebastian Conrad (Hg.) (2010): Mission und kulturelle Globalisierung, Geschichte und Gesellschaft 36/2 (Themenheft).

Harders, Levke (2014): Legitimizing Biography: Critical Approaches to Biographical Research, in: GHI Bulletin 55, 49-56.

Harzig, Christiane, Dirk Hoerder und Donna R. Gabaccia (2009): What Is Migration History? Cambridge.

Hausberger, Bernd (2006): Globale Lebensläufe: Menschen als Akteure im weltgeschichtlichen Geschehen, Wien.

Herren, Madeleine (2005): Die Inszenierung des globalen Subjekts. Vorschläge zur Typologie einer transgressiven Biographie, in: Historische Anthropologie 13, 1-18.

Herren, Madeleine (2009): Internationale Organisationen seit 1865: eine Globalgeschichte der internationalen Ordnung, Darmstadt.

Hilton, Matthew und Rana Mitter (Hg.) (2013): Transnationalism and Contemporary Global History, Oxford.

Hochgeschwender, Michael (1998): Freiheit in der Offensive? Der Kongress für Kulturelle Freiheit und die Deutschen, München.

Hoerder, Dirk (2002): Cultures in Contact: World Migrations in the Second Millennium, Durham.

Hoerder, Dirk (2005): Transkulturelle Lebensformen. Menschen in lokalen - (post-)nationalen - globalen Welten, in: Sozial.Geschichte 20, 11-29.

Horne, Julia (2010): The Cosmopolitan Life of Alice Erh-Soon Tay, in: Journal of World History 21, 419-446.

Iriye, Akira und Pierre-Yves Saunier (Hg.) (2009): The Palgrave Dictionary of Transnational History, Basingstoke.

James, Kathleen (2006): Bauhaus Culture: From Weimar to the Cold War, Minneapolis.

Klein, Christian (2009): Handbuch Biographie. Methoden, Traditionen, Theorien, Stuttgart.

Klimke, Martin (2010): The Other Alliance: Student Protest in West Germany and the United States in the Global Sixties, Princeton. 
Köhler, Ingo und Roman Rossfeld (Hg.) (2012): Pleitiers und Bankrotteure. Geschichte des ökonomischen Scheiterns vom 18. bis zum 20. Jahrhundert, Frankfurt am Main.

Krauss, Marita (2001): Heimkehr in ein fremdes Land: Geschichte der Remigration nach 1945, München.

Krige, John und Helke Rausch (Hg.) (2012): American Foundations and the Coproduction of World Order in the Twentieth Century, Göttingen.

Krohn, Claus-Dieter (1988): Vereinigte Staaten von Amerika, in: Claus-Dieter Krohn et al. (Hg.): Handbuch der deutschsprachigen Emigration, Darmstadt.

Krohn, Claus-Dieter (Hg.) (2009): Exil, Entwurzelung, Hybridität (=Jahrbuch Exilforschung 27), München.

Langenbucher, Wolfgang (Hg.) (2008): Paul Felix Lazarsfeld: Leben und Werk. Anstatt einer Biografie, Wien.

Lässig, Simone (2012): Übersetzung in der Geschichte - Geschichte als Übersetzung? Überlegungen zu einem analytischen Konzept und Forschungsgegenstand für die Geschichtswissenschaft, in: Geschichte und Gesellschaft 38, 189-216.

Leman, Merel (2014): The Transatlantic Reconstruction of Western Culture: George Mosse and Peter Gay, in: Jan Logemann und Mary Nolan (Hg.) (2014): More Atlantic Crossings? European Voices and the Postwar Atlantic Community, (GHI Bulletin Supplement 10), Washington, 139-160.

Logemann, Jan, Andreas Joch, Corinna Ludwig, Ashley Narayan und Barbara Reiterer (2011): Transatlantic Perspectives: Europe in the Eyes of European Immigrants to the United States, 1930-1980, in: GHI Bulletin 48, 85-99.

Logemann, Jan (2013): European Imports? European Immigrants and the Transformation of American Consumer Culture from the 1920s to the 1960s, in: GHI Bulletin 52, 113-133.

Logemann, Jan und Mary Nolan (Hg.) (2014): More Atlantic Crossings? European Voices and the Postwar Atlantic Community, (GHI Bulletin Supplement 10), Washington.

Logemann, Jan, Donna Gabaccia und Sally Gregory Kohlstedt (Hg.) (2014): Europe, Migration and Identity: Connecting Migration Experiences and Europeanness, London.

Louis, Barbara (2013): Gender and Identity in Exile: A European émigré in Social Work, in: National Identities 15, 51-66.

Lubinski, Christina, Jeffrey R. Fear und Paloma Fernández Pérez (2013): Family Multinationals: Entrepreneurship, Governance, and Pathways to Internationalization, New York.

Lüthi, Barbara und Miriam Rürup (2010): Grenzgänge [Editorial zu einem Sonderheft], in: WerkstattGeschichte 53.

McKeown, Adam (2008): Melancholy Order: Asian Migration and the Globalization of Borders, New York.

Middell, Matthias (2000): Kulturtransfer und Historische Komparatistik - Thesen zu ihrem Verhältnis, in: Comparativ 10, 7-41.

Milder, Stephen (2010): Thinking Globally, Acting (Trans-)Locally: Petra Kelly and the Transnational Roots of West German Green Politics, in: Central European History 43, 301326.

Mittag, Jürgen und Berthold Unfried (2008): Transnationale Netzwerke - Annäherungen an ein Medium des Transfers und der Machtausübung, in: Berthold Unfried et al. (Hg): Transnationale Netzwerke im 20. Jahrhundert, Wien, 9-25.

Neurath, Wolfgang und Lothar Krempel (2008): Geschichtswissenschaft und Netzwerkanalyse: Potenziale und Beispiel, in: Berthold Unfried et al. (Hg): Transnationale Netzwerke im 20. Jahrhundert, Wien, 59-79.

Nolan, Mary (2012): The Transatlantic Century: Europe and America, 1890-2010, Cambridge.

Osterhammel, Jürgen (2009): Die Verwandlung der Welt: eine Geschichte des 19. Jahrhunderts, München.

Osterhammel, Jürgen und Niels Petersson ( $\left.{ }^{5} 2012\right)$ : Geschichte der Globalisierung: Dimensionen, Prozesse, Epochen, München, 7-27. 
Patel, Kiran Klaus (2003): Transatlantische Perspektiven transnationaler Geschichte, in: Geschichte und Gesellschaft 29, 625-647.

Pertilla, Atiba und Uwe Spiekermann (2014): Living the American Dream? The Challenge of Writing Biographies of German-American Immigrant Entrepreneurs, in: GHI Bulletin $55,77-90$.

Portes, Alejandro (2000): Globalization from Below: The Rise of Transnational Communities, in: Don Kalb u.a. (Hg.): The Ends of Globalization, Oxford, 253-270.

Rischbieter, Laura (2011): Mikro-Ökonomie der Globalisierung: Kaffee, Kaufleute und Konsumenten im Kaiserreich 1870-1914, Köln.

Rodgers, Daniel (1998): Atlantic Crossings: Social Politics in a Progressive Age. Cambridge.

Rodogno, Davide, Bernhard Struck und Jakob Vogel (Hg.) (2015): Shaping the Transnational Sphere: Experts, Networks and Issues from the 1840s to the 1930s, New York.

Rosenberg, Emily S. (2012): A World Connecting 1870-1945, Cambridge.

Schmelzer, Matthias (2012): The Crisis before the Crisis: The 'Problems of Modern Society' and the OECD, 1968-1974, in: European Review of History 19/6, 999-1020.

Schweiger, Hannes (2012): Global Subjects: The Transnationalisation of Biography, in: Life Writing 9, 249-258.

Scott-Smith, Giles (2010): The Congress for Cultural Freedom: Constructing an Intellectual Atlantic Community, in: Michael Mariano (Hg.): Defining the Atlantic Community: Culture, Intellectuals, and Policies in the Mid-Twentieth Century, London, 132-145.

Shaw, Lauren (2014): Migrants as "Translators": Mediating External Influences on Post World War II Western Europe (Konferenzbericht), in: GHI Bulletin 54, 125-130.

Slobodian, Quinn (2012): Foreign Front: Third World Politics in Sixties West Germany, Durham.

Slobodian, Quinn (2014): The World Economy and the Color Line: Wilhelm Röpcke, Apartheid and the White Atlantic, in: Jan Logemann und Mary Nolan (Hg.) (2014): More Atlantic Crossings? European Voices and the Postwar Atlantic Community, (GHI Bulletin Supplement 10), Washington, 61-89.

Sluga, Glenda und Julia Horne (2010): Cosmopolitanism: Its Pasts and its Practices, in: Journal of World History 21, 369-374.

The German Historical Institute at 25 Years, GHI Bulletin Supplement 8 (2012).

Thelen, David (1992): Of Audiences, Borderlands and Comparisons: Towards the Internationalization of American History, in: Journal of American History 79, 432-462.

Torp, Cornelius (2005): Die Herausforderung der Globalisierung: Wirtschaft und Politik in Deutschland 1860-1914, Göttingen.

Tyrrell, Ian (1991): American Exceptionalism in an Age of International History, in: American Historical Review 96, 1031-1055.

Tyrrell, Ian (2007): Transnational Nation: United States History in Global Perspective Since 1789, Basingstoke.

Tyrrell, Ian (2009): Reflections on the Transnational Turn in United States History: Theory and Practice, in: Journal of Global History 3, 453-474.

Unger, Corinna (2009): Reise ohne Wiederkehr? Leben im Exil 1933 bis 1945, Darmstadt.

Wagner, Phillip (2014): New Life for American Downtowns? The 1958 International Seminar on Urban Renewal and the Travel of Planning Ideas in the North Atlantic World, in: Planning Perspectives 29, 189-208.

Wheatland, Thomas und Franz L. Neumann (2014): Negotiating Political Exile, in: Jan Logemann und Mary Nolan (Hg.) (2014): More Atlantic Crossings? European Voices and the Postwar Atlantic Community, (GHI Bulletin Supplement 10), Washington, 111138.

Zeisel, Hans (1988): Die Wiener Schule der Motivforschung, in: Joseph Langer (Hg.), Geschichte der österreichischen Soziologie, Wien, 157-166. 


\section{Zusammenfassung}

Transnationale Biographien eröffnen wichtige Perspektiven für eine zunehmend global orientierte Geschichtswissenschaft. Am Beispiel der transatlantischen Karrieren von Paul Lazarsfeld und anderen europäischen Emigranten in den Vereinigten Staaten von Amerika untersucht dieser Aufsatz, inwiefern der biographische Zugriff unser Verständnis für die Mikroebene transnationaler Transferprozesse schärfen kann.

Transnationale Biographik hat zahlreiche Anknüpfungspunkte in der neueren Migrations- und Transferforschung sowie in der Globalgeschichte. Kosmopolite Lebensläufe fanden hier bisher vor allem als ,transgressive Biographien“ oder als „transnationale Mittler" Beachtung.

Aufbauend auf den Ergebnissen des Forschungsprojekts Transatlantic Perspectives am Deutschen Historischen Institut in Washington wird die Bedeutung von ,transnationalen Mittlern" für drei zentrale Aspekte von Transfer- und Austauschprozessen herausgearbeitet: 1. die Bedeutung von Migranten für grenzüberschreitenden und reziproken Wissenstransfer, 2. die Bedeutung von Netzwerken für die Verstetigung und Nachhaltigkeit von Austauschprozessen, sowie 3. die Zentralität von aktiven Übersetzungsleistungen bei der Lokalisierung und Adaption von Wissen und Praktiken. Abschließend werden auch blinde Flecken eines solchen Zugangs zu transnationaler Geschichte kurz problematisiert. 